\title{
Growth and physico chemical characterization of lanthanum neodymium oxalate single crystals
}

\author{
K S RAJU*, VARUGHESE JOHN ${ }^{\dagger}$ and M A ITTYACHEN ${ }^{\dagger}$ \\ Department of Crystallography and Biophysics, University of Madras, Guindy Campus, Madras 600025 , India \\ ${ }^{\dagger}$ School of Pure and Applied Physics, Mahatma Gandhi University, Kottayam 686031, India \\ MS received 26 March 1998; revised 1 July 1998
}

\begin{abstract}
Single crystals of lanthanum neodymium oxalate (LNO) are grown in sodium meta silicate gels, by the diffusion of a mixture of aqueous solutions of lanthanum nitrate and neodymium nitrate into the test tube having the set gel containing oxalic acid. The bluish pink coloured tabular crystals of LNO having well defined hexagonal basal planes appear either as 'foggy' or 'clear', the latter at the greater depths inside the gel. The colouration of LNO visually observed is evidenced in UV-visible spectrum, by the revelation of well pronounced characteristic peaks in the visible region (500-900 nm). X-ray diffraction (XRD) of powdered LNO is ordered, meaning crystalline in nature, besides its 'isostructurality' with similarly grown lanthanum samarium oxalate crystals. The single crystallinity of LNO is established by its oscillation XRD pattern. Thermogravimetric analysis (TGA) and differential scanning colorimetry (DSC) support that LNO loses water of crystallization around $120^{\circ} \mathrm{C}$ and $\mathrm{CO}$ and $\mathrm{CO}$, around $350-450^{\circ} \mathrm{C}$, while the infrared absorption (IR) spectrum of $\mathrm{LNO}$ establishes the presence of oxalate $\left(\mathrm{C}_{2} \mathrm{O}_{4}\right)^{2-}$ ions. Energy dispersive X-ray analysis (EDAX) confirms the presence of La and Nd in the sample. X-ray photoelectron spectroscopic (XPS) studies of LNO establish the presence of La and Nd in their respective oxide states. An empirical structure for LNO has been proposed on the basis of these findings. The 'smokiness' in the foggy LNO crystal has been attributed due to the 'gel inclusion' during the growth process.
\end{abstract}

Keywords. Lanthanum neodymium oxalate crystals; growth; UV-visible; XRD; TGA; DSC; IR; EDAX; XPS.

\section{Introduction}

The importance of rare earth compounds has gained recognition of materials scientists for their interesting luminescent and magnetic properties (Eyring 1964). The ferroelectric and ferroelastic properties of rare earth molybdates and oxalates find applications in electro and acousto optical devices (Barkley et al 1971, 1972; Sapriel and Vacher 1977). Synthesis of superconducting compounds by the controlled precipitation of oxalates followed by calcination has been reported (Caillaud et al 1988). Beille et al (1987) have shown that lanthanum copper oxide exhibits superconductivity up to $40 \mathrm{~K}$. Barium oxalate tetragonal bipyramidal crystals are grown by the precipitation of alkaline earth metal oxalate powders from the neutral aqueous solution (Pacter 1977). At elevated temperatures, rare earth oxalates are grown by employing Czochralski technique (Brixner 1973), which imbibe thermal defects. In order to avoid these defects, methods of growth at room temperature are preferred. Sparingly soluble inorganic crystals are grown by the well established gel technique pioneered by Henisch et al (1965) and Henisch (1970) despite the limitations in the sizes of the gel grown crystals.

*Author for correspondence
The rare earth mixed oxalate and molybdate crystals are grown by gel method at room temperature and characterized employing several techniques (Raju et al 1994; Jayakumari et al 1995; Sushma Bhat et al 1995). The authors report here the work carried out on the growth of LNO in gels, besides their characterization employing UV-visible, XRD, TGA, IR, EDAX and XPS techniques, which sheds important results in understanding the kinetics of chemical reactions, thermal stability, the detection of heavy elements present in the mixed oxalates, as well as identification of their chemical states. The empirical structure of LNO sample has been proposed on the basis of these findings.

\section{Experimental and results}

\subsection{Growth of LNO in gels}

Aqueous solution of sodium meta silicate (specific gravity: $1.04 \mathrm{~g} \mathrm{~cm}^{-3}$ ) is adjusted to the desired $\mathrm{pH}$ by adding analytical grade nitric acid and used as the reacting medium in a test tube (35 $\mathrm{mm}$ dia and $200 \mathrm{~mm}$ in length). A mixture of aqueous solutions of lanthanum nitrate and neodymium nitrate with nitric acid is used as the outer 
reactant above the gel at room temperature. The outer reactant diffuses into the set gel embedded with oxalic acid and the following reaction takes place producing bluish pink colloidal precipitate of mixed oxalates of lanthanum and neodymium $\left[\mathrm{La} \cdot \mathrm{Nd}\left(\mathrm{C}_{2} \mathrm{O}_{4}\right)_{3}\right]$ and nitric acid.

$$
\left.\begin{array}{c}
\mathrm{La}\left(\mathrm{NO}_{3}\right)_{3} \\
+ \\
\mathrm{Nd}\left(\mathrm{NO}_{3}\right)_{3}
\end{array}\right]+\begin{aligned}
& 3 \mathrm{H}_{2} \mathrm{C}_{2} \mathrm{O}_{4} \rightarrow \\
& \mathrm{La} \cdot \mathrm{Nd}\left(\mathrm{C}_{2} \mathrm{O}_{4}\right)_{3} \cdot n \mathrm{H}_{2} \mathrm{O}+6 \mathrm{HNO}_{3}
\end{aligned}
$$

The proposed empirical structure of lanthanum neodymium oxalate is given in figure 1 . The bluish pink coloured crystals of LNO nucleating from the precipitate are well defined isolated tabular crystals with the hexagonal basal planes and are found to be either 'foggy' (figure 2a) or 'clear' (figure 2b). The 'foggy' crystals are formed closer to the precipitate layer inside the gel, while 'clear' crystals of relatively bigger sizes are observed at the greater depths inside the gel (average size after four weeks: $4 \times 3 \times 2 \mathrm{~mm}^{3}$ ). For the growth of good individual crystals of LNO, the optimum conditions are found to be at $\mathrm{pH}$ of 6 of the gel impregnated before setting with $1 \mathrm{M}$ oxalic acid and the mixture of each of the rare earth nitrates $(1: 1)$ by volume as the outer reactant.

\subsection{Characterization of $L N O$}

2.2a Experimental: The UV-visible absorption spectrum of LNO is recorded in Jasco UV-visible spectrometer mode No. (V-530), Japan. The X-ray diffraction patterns of oscillation and powdered LNO sample are taken in a Philips XRD PW $1050 / 70$ with $\mathrm{CuK}_{\alpha}$ radiation

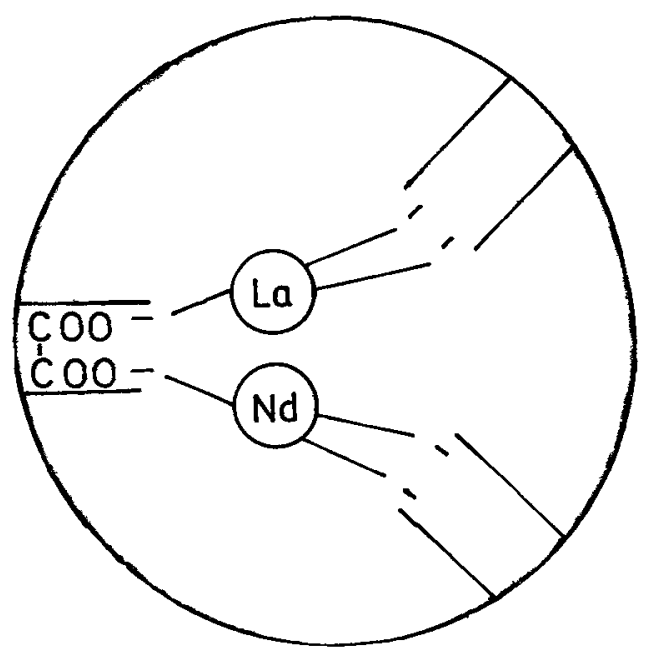

Figure 1. The proposed empirical structure of LNO. $(\lambda=1.5418 \AA)$. The TGA/DSC plots of LNO crystal are recorded using Delta series TGA 7 , at the scanning of $20^{\circ} \mathrm{C} / \mathrm{min}$. The IR absorption spectrum of LNO sample taken in $\mathrm{KBr}$ matrix, is recorded in IR-PERKIN ELMER model 350, England. The sample preparation for EDAX and ESCA is as follows. LNO is finely powdered and pellets of $1 \mathrm{~cm}$ dia and $1 \mathrm{~mm}$ thickness are formed using a hydraulic press by applying a pressure of $1 \cdot 1$ tons $/ \mathrm{cm}^{2}$. For EDAX, the surface of LNO pellet mounted onto an aluminium stub is gold coated so as to make a good electrical contact and is examined in the EDAX analyzer No. 711 , an accessory to the scanning electron microscope, Philip's SEM model 501. XPS spectra of LNO pellet are recorded in VG scientific ESCALAB MK II, by irradiating the sample with the monochromatic soft $\mathrm{X}$-rays $\left(\mathrm{MgK}_{\alpha}\right.$; photon energy $\left.1253.6 \mathrm{eV}\right)$. The constant analyzer is $50 \mathrm{eV}$ and the resolution of the system is about $0.7 \mathrm{eV}$.

$2.2 \mathrm{~b} U V$-visible spectral studies: It is observed in the UV-visible spectrum of LNO (figure 3 ) that the strong intense peak around $250 \mathrm{~nm}$ is assigned to the oxalate
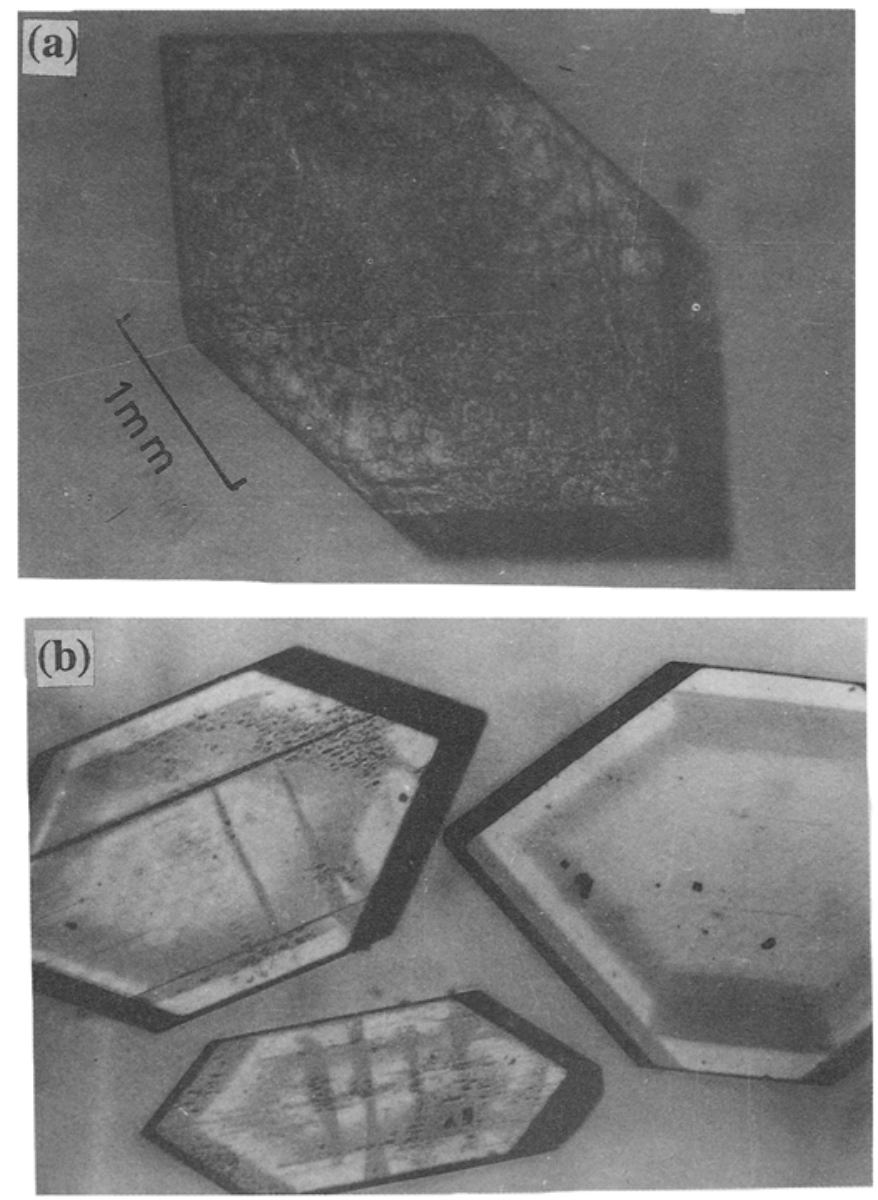

Figure 2. SEM pictures of a. foggy LNO crystal $(\times 50)$ and b. clear LNO crystals. 
group, as this strong peak in position (nm) and intensity is identified in the UV-visible spectrum of oxalic acid solution. The small peak (marked by an arrow) next to oxalate peak positioned at $300 \mathrm{~nm}$ is attributed to lanthanum in the UV region, as the same is revealed in the UV-visible spectrum of lanthanum nitrate. The remaining peaks in the visible region labelled neodymium under brackets, match exactly with the UV-visible peaks of neodymium in the literature (Huheey 1983). It may be worth mentioning that the peaks positioned in the visible region (from 500 to $900 \mathrm{~nm}$ ) account well for crystal having bluish pink colouration.

2.2c XRD studies: The well defined Bragg peaks in the XRD of the powdered LNO at specific ' $2 \theta$ ' angles indicate that LNO sample is ordered (figure 4a). The Bragg peaks in the XRD pattern of powdered LNO (figure 4a) have a reasonable agreement with the Bragg peaks in the XRD pattern of powdered lanthanum samarium oxalate (figure 4b), meaning both are isostructural'. The oscillation XRD pattern of LNO reveals well defined Bragg reflections at specific ' $2 \theta$ ' angles (figure 5) giving the strong clue that LNO is single crystalline in nature.

2.2d TGA and DSC of LNO: TGA and DSC plots of LNO (figure 6) show that the loss of weight in the temperature range around $100^{\circ} \mathrm{C}$ relates to the loss of water of crystallization (endothermic reaction) (see (1) below). The loss of weight around $420^{\circ} \mathrm{C}$ has two chemical changes, one relating to release of $\mathrm{CO}$ at $400^{\circ} \mathrm{C}$ and the other at $430^{\circ} \mathrm{C}$ relating to $\mathrm{CO}_{2}$, which are exothermic in character and very close to each other (see (2)).

$$
\begin{aligned}
& \mathrm{La} \cdot \mathrm{Nd}\left(\mathrm{C}_{2} \mathrm{O}_{4}\right)_{3} \cdot n \mathrm{H}_{2} \mathrm{O} \rightarrow \mathrm{La} \cdot \mathrm{Nd}\left(\mathrm{C}_{2} \mathrm{O}_{4}\right)_{3}+n \mathrm{H}_{2} \mathrm{O}, \\
& \mathrm{La} \cdot \mathrm{Nd}\left(\mathrm{C}_{2} \mathrm{O}_{4}\right)_{3} \rightarrow \mathrm{La} \cdot \mathrm{Nd} \mathrm{O}_{3}(s) \\
& +3 \mathrm{CO}(g)+3 \mathrm{CO}_{2}(g) .
\end{aligned}
$$

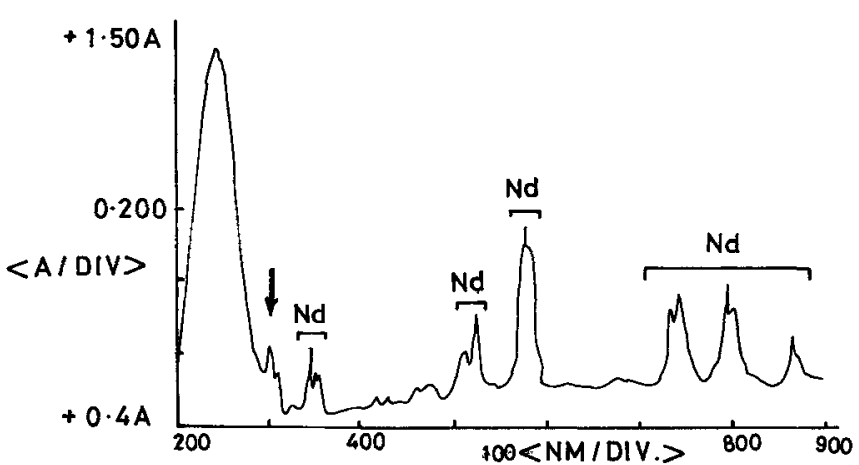

Figure 3. UV-visible spectrum of LNO.
(La. Nd) $\mathrm{O}_{3}$ is fairly stable and the residue mainly consists of this compound beyond $620^{\circ} \mathrm{C}$.

2.2 $\mathrm{e}$ IR absorption studies: In the IR absorption spectrum of LNO (figure 7), the presence of water of crystallization is established by the revelation of well pronounced broad intense peak at $3300 \mathrm{~cm}^{-1}$ relating to asymmetric and symmetric $\mathrm{OH}$ stretchings and the peak at $1630 \mathrm{~cm}^{-1}$ relating to $\mathrm{HOH}$ bending plus $\gamma_{\mathrm{a}}(\mathrm{C}=\mathrm{O})$. The peaks positioned at $1380 \mathrm{~cm}^{-1}$ and the more intense at $1320 \mathrm{~cm}^{-1}$ are attributed to the symmetric stretchings of $\mathrm{CO}_{2}$. The sharp peak at $810 \mathrm{~cm}^{-1}$ is due to the combined effects of in-plane deformation of $\mathrm{CO}_{2}$ and the presence of metal-oxygen bonds. The rocking of $\mathrm{CO}_{2}$ is related to the medium peak at $480 \mathrm{~cm}^{-1}$.

2.2f EDAX of LNO: In order to establish the elemental incorporation like $\mathrm{La}$ and $\mathrm{Nd}$ in LNO, EDAX work has been taken up. In the EDAX pattern of LNO sample (figure $8 \mathrm{a}$ ), the three peaks positioned at $4.65,5.04$ and $5.38 \mathrm{keV}$ relate to $L_{\alpha}, L_{\beta 1}$ and $L_{\beta 2}$ energies of lanthanum, whereas $L_{\alpha}, L_{\beta 1}$ and $L_{\beta 2}$ energies relating to neodymium are represented by peaks positioned at $5.23,5.72$ and $6.088 \mathrm{keV}$, respectively. The energy values $(\mathrm{keV})$ reported for $\mathrm{La}\left(L_{\alpha, \beta 1, \beta 2}\right)$ and $\mathrm{Nd}\left(L_{\alpha, \beta 1, \beta 2}\right)$ in the present $\operatorname{EDAX}$ investigation are in excellent agreement with the EDAX library.

2.2g XPS studies: XPS studies are undertaken in order to re-establish the constituents incorporated in LNO sample besides their chemical states. The XPS peaks relating to $\mathrm{La}\left(3 d_{5 / 2}-3 d_{3 / 2}\right)$ show binding energy values centred around $835 \mathrm{eV}$ and $852 \mathrm{eV}$ with the separation of $16.8 \mathrm{eV}$ (figure 9), while figure 10 relates to the XPS peak of $\mathrm{Nd}\left(3 d_{5 / 2}\right)$ having the binding energy value at $980 \mathrm{eV}$. The XPS region of $\mathrm{C}_{1 \mathrm{~s}}$ of LSO sample (figure 11) shows two peaks having binding energies around

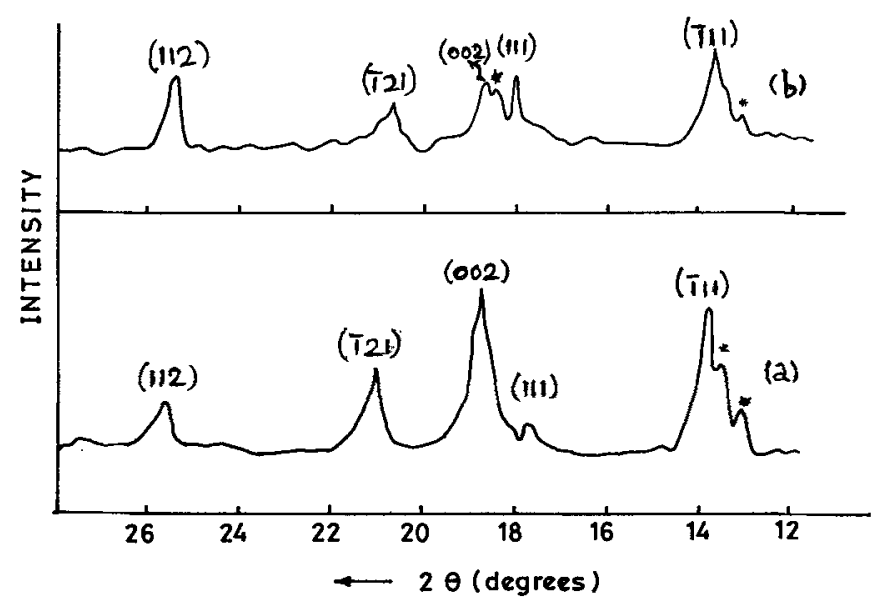

Figure 4. X-ray diffraction patterns of (a) powdered LNO and (b) powdered lanthanum samarium oxalate. 


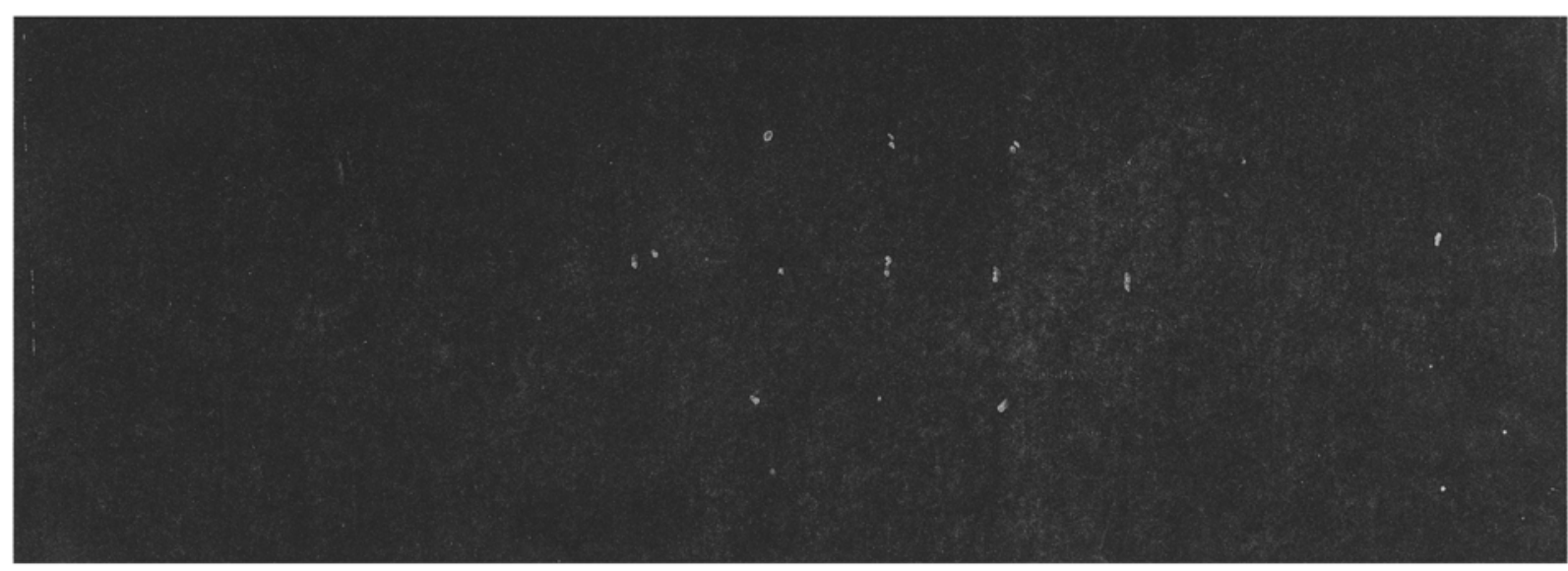

Figure 5. Oscillation X-ray diffraction pattern of LNO.

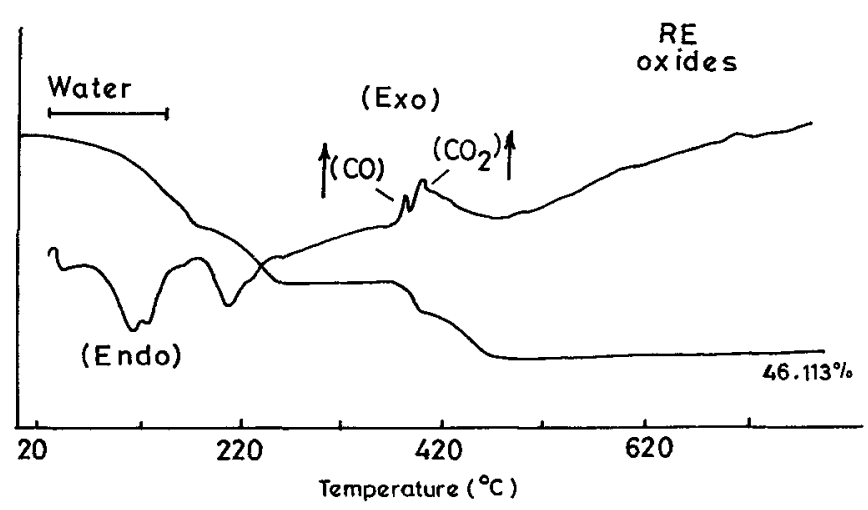

Figure 6. TGA/DSC plots of LNO.

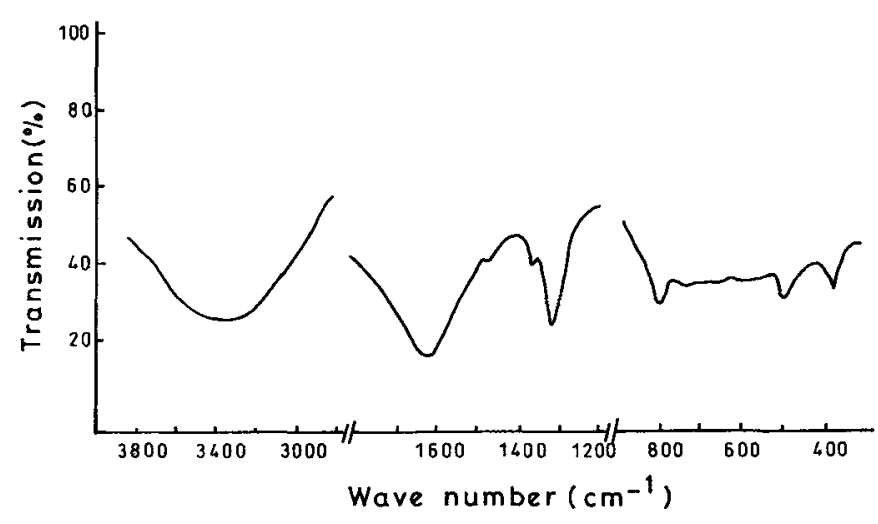

Figure 7. IR absorption spectrum of LNO.

287 and $290 \mathrm{eV}$. The first peak positioned at $287 \mathrm{eV}$, is attributed to the adventitious hydrocarbon nearly always present from the laboratory environment or from the glove box, while the second peak at the higher binding energy value of $290 \mathrm{eV}$ relates to the oxalate $\left(\mathrm{C}_{2} \mathrm{O}_{4}\right)$ group. The second peak relating to the oxalate group is shifted with respect to the first peak towards the higher
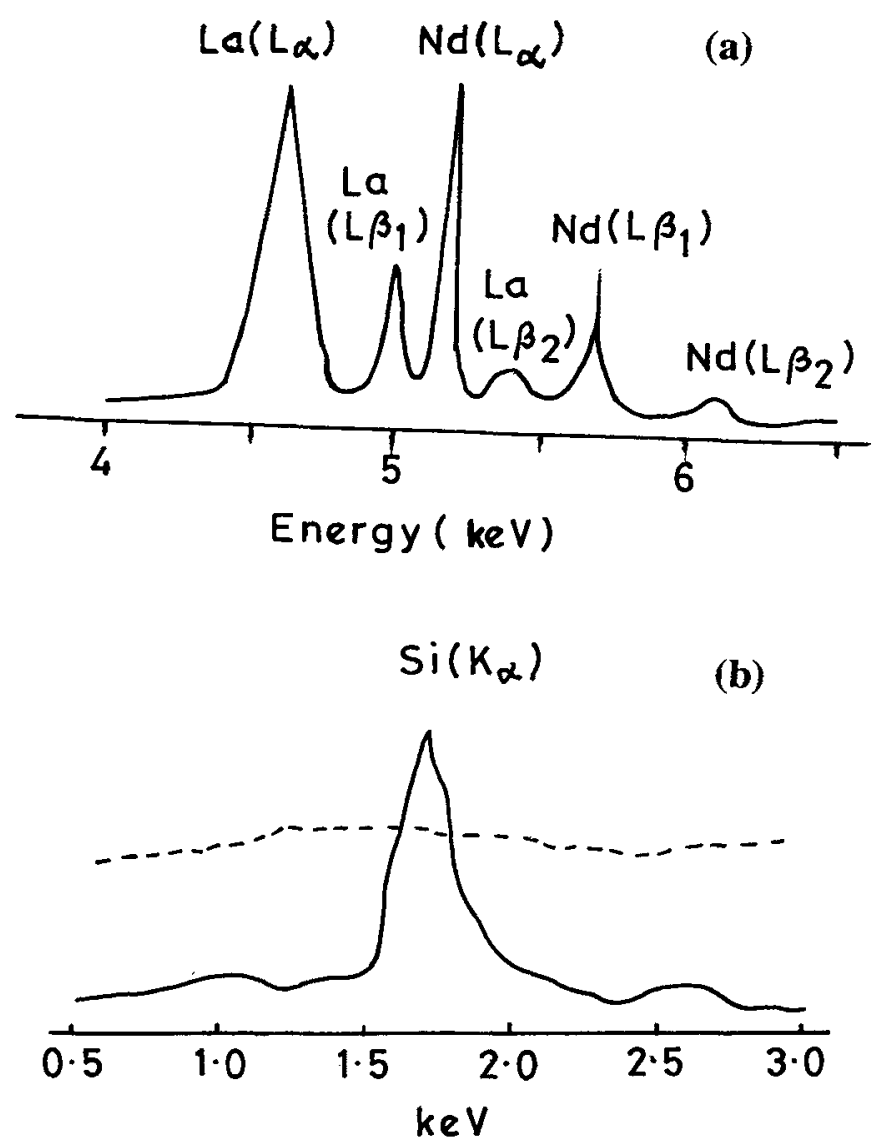

Figure 8. a. EDAX pattern of LNO and b. EDAX pattern of $\mathrm{Si}\left(K_{\alpha}\right)$ region relating to foggy LNO crystal (continuous line) and of clear LNO crystal (dashed line).

binding energy value and this is attributed due to higher electronegativity of oxalate group. The binding energy values relating to $\mathrm{La}\left(3 d_{5 / 2}-3 d_{3 / 2}\right)$ and $\mathrm{Nd}\left(3 d_{5 / 2}\right)$ along with their separations (figures 9-10) agree quite well with the literature values (Wagner et al 1978) corresponding 
to their oxide states $\left(\mathrm{La}_{2} \mathrm{O}_{3}\right.$ and $\left.\mathrm{Nd}_{2} \mathrm{O}_{3}\right)$. It is well known that the XPS, though a surface analysis, is a powerful technique for the identification of constituent elements present in the sample besides their respective chemical states.

$2.2 \mathrm{~h}$ 'Foggy' crystal studies: In order to analyze the 'smokiness' of the 'foggy crystals', EDAX spectrum is taken for a foggy LNO crystal (solid line in figure 8b) which reveals a weak peak magnified, positioned at the binding energy $1.73 \mathrm{keV}$. This energy value relates well to $\operatorname{Si}\left(K_{\alpha}\right)$, which undoubtedly is the contribution of the gel medium. In order to confirm this, EDAX pattern of 'clear' LNO crystal has been recorded in the region of $\mathrm{Si}\left(K_{\alpha}\right)$ (dashed line in figure $8 \mathrm{~b}$ ), in which it is obviously evident that the peak relating to $\mathrm{Si}\left(K_{a}\right)$ at $1.73 \mathrm{keV}$ is 'absent', meaning thereby 'gel inclusion' is 'nil' or

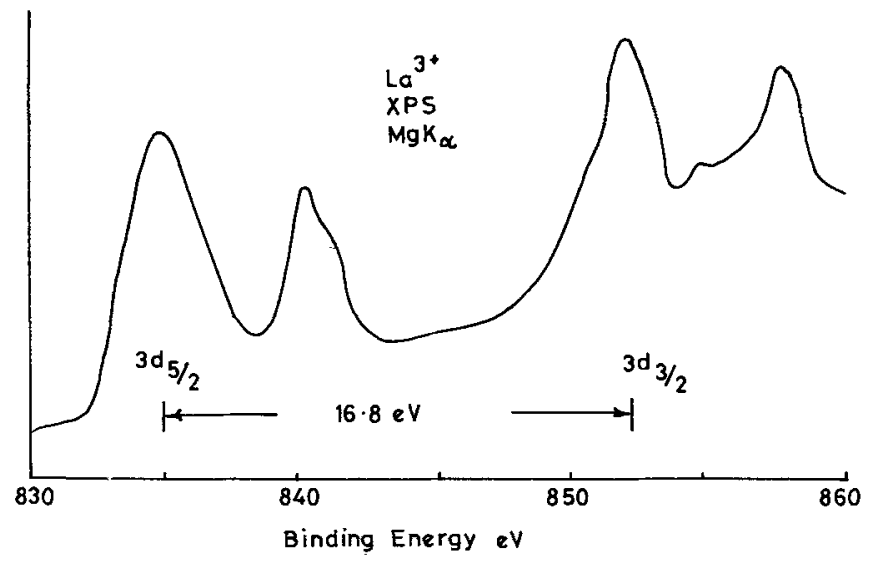

Figure 9. XPS peaks of $\mathrm{La}\left(3 d_{5 / 2}-3 d_{3 / 2}\right)$ of LNO.

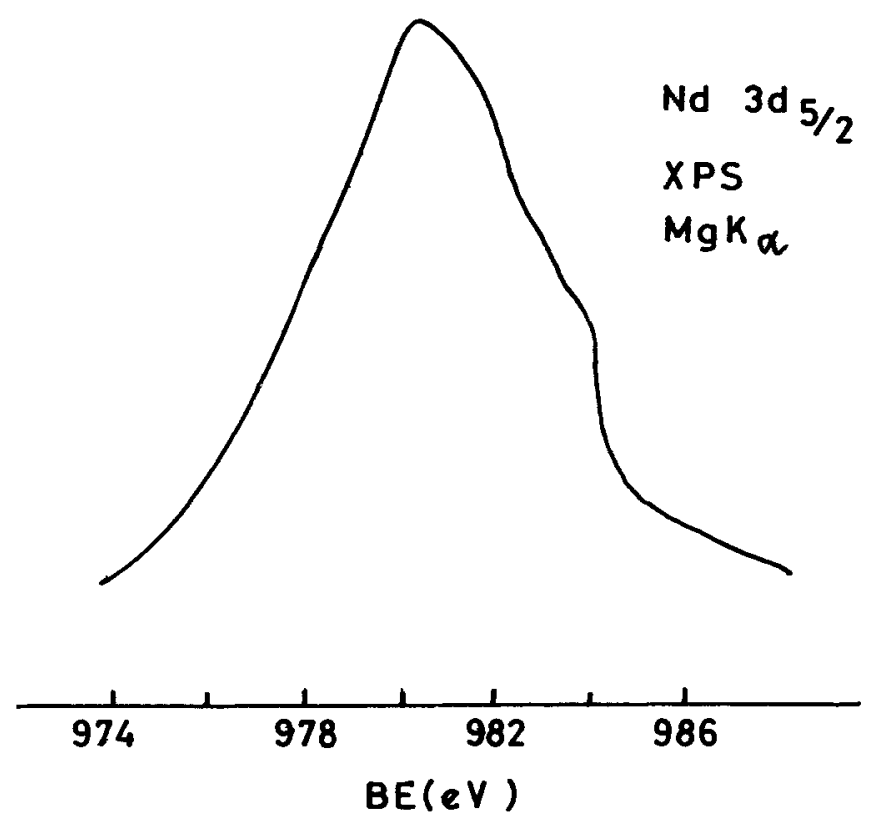

Figure 10. XPS peak of $\mathrm{Nd}\left(3 d_{5 / 2}\right)$ of LNO. 'negligibly small' in the case of 'clear' LNO crystal. It is indeed interesting that the XPS of foggy crystal (figure 12) exhibits a weak peak magnified, positioned at the binding energy $103.4 \mathrm{eV}$, which relates quite well to the $(2 p)$ states of $\mathrm{SiO}_{2}$. The chemical state of $\mathrm{Si}$ in the present case as ' $\mathrm{SiO}_{2}$ ' is understandable, for sodium meta silicate $\left(\mathrm{Na}_{2} \mathrm{SiO}_{3}\right)$ may be considered as the combination of $\mathrm{Na}_{2} \mathrm{O}$ and $\mathrm{SiO}_{2}$. Thus the 'smokiness' of the 'foggy' crystal may be attributed to the 'gel inclusion' in the LNO crystal during the growth process.

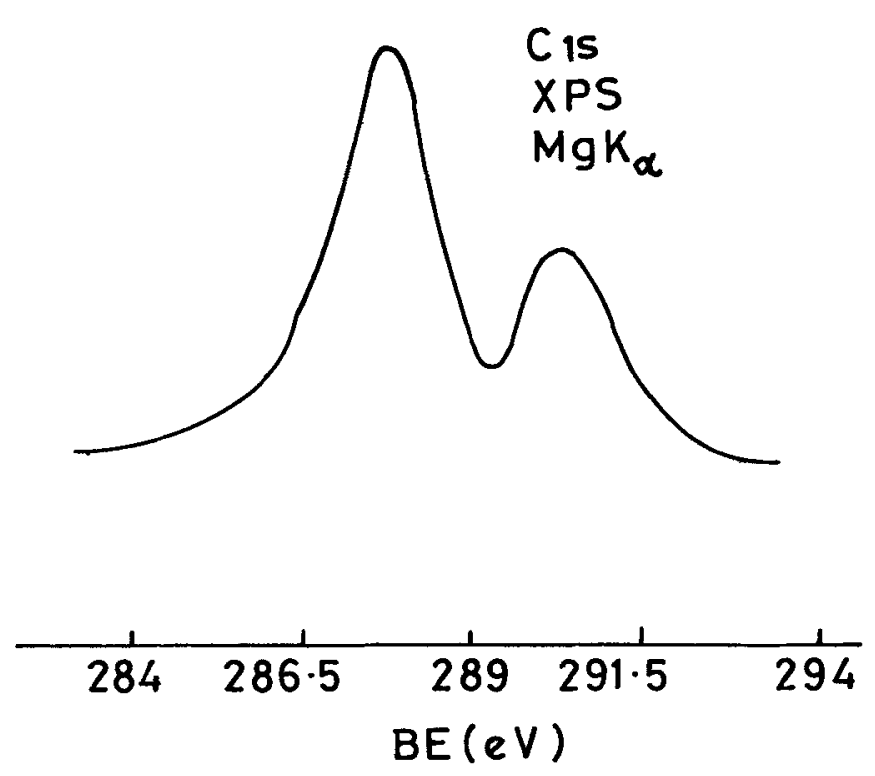

Figure 11. XPS peaks of $\mathrm{C}_{1 \mathrm{~s}}$ of LNO.

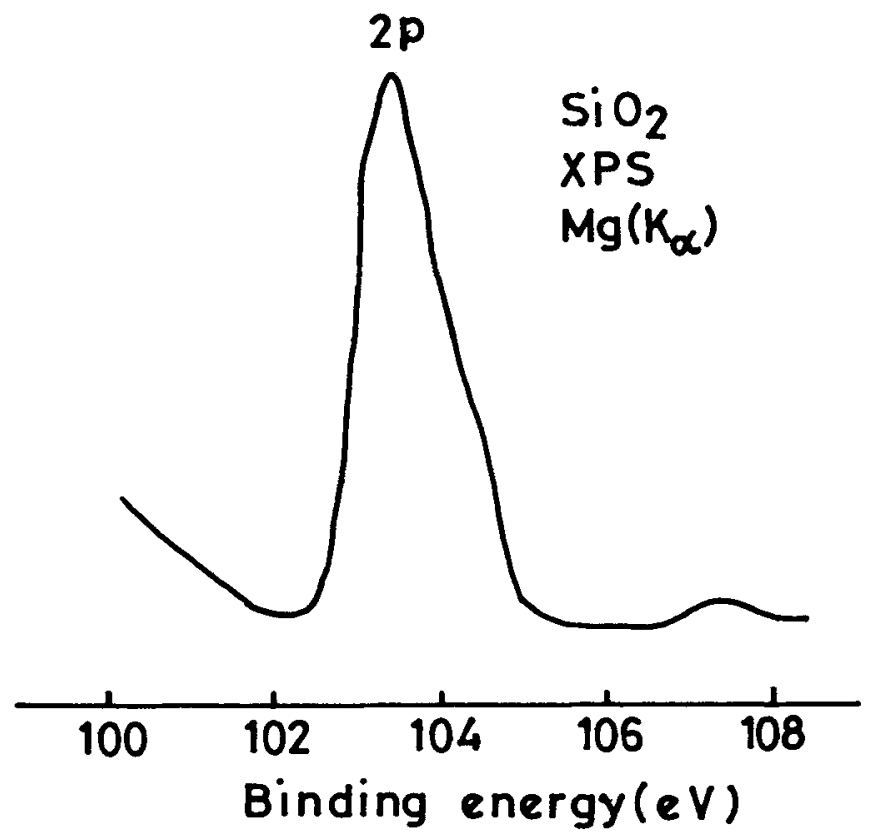

Figure 12. XPS peaks of $2 p$ levels of $\mathrm{SiO}_{2}$. 


\section{Discussion}

In the present work, the gel grown LNO is crystalline in nature, is obviously evident by the revelation of well defined edges and corners with the hexagonal basal planes observed at varying depths in the gel (figures 2a and $b$ ). The crystals of LNO are bluish pink in colour and transparent, are also indicated by the presence of absorption peaks in the visible region of the UV-visible spectrum of LNO (figure 3 ). The crystalline nature of LNO is evidenced by the revelation of well defined Bragg peaks at specific ' $2 \theta$ ' angles in the XRD pattern of powdered sample (figure $4 \mathrm{a}$ ) which is shown to be 'isostructural' on comparison with the XRD of powdered lanthanum samarium oxalate, grown under identical conditions as LNO (compare figures $4 \mathrm{a}$ and $\mathrm{b}$ ). The Bragg peaks unindexed (marked by asterisk), in the XRD of powdered samples of LNO and lanthanum samarium oxalate (figures $4 \mathrm{a}$ and $\mathrm{b}$ ) may be attributed to the presence of impurities incorporated during crystal growth. That LNO is single crystalline, is evidenced by the revelation of well defined Bragg reflections at specific ' $2 \theta$ ' angles in the oscillation XRD pattern of LNO sample (figure 5). TGA of LNO (figure 6) reveals that the crystals lose water of crystallization around 100$200^{\circ} \mathrm{C}$ and $\mathrm{CO}$ and $\mathrm{CO}_{2}$ around $400-420^{\circ} \mathrm{C}$ and the residue beyond $500^{\circ} \mathrm{C}$ being $\mathrm{La}$ and $\mathrm{Nd}$ oxides $(46.113 \%)$. The IR absorption spectrum of LNO (figure 7) establishes the presence of oxalate complex $\left(\mathrm{C}_{2} \mathrm{O}_{4}\right)^{2-}$ in the sample. The binding energy values of the EDAX peaks (figure 8) relating to $\mathrm{La}\left(L_{\alpha}, L_{\beta 1,2}\right), \mathrm{Nd}\left(L_{\alpha}, L_{\beta 1,2}\right)$ and $\operatorname{Si}\left(K_{\alpha}\right)$ agree quite well with the literature values (EDAX Library), thereby confirming the incorporation of $\mathrm{La}$ and $\mathrm{Nd}$ in LNO, besides 'gel inclusion' if any. The heights and areas (Riggs and Parker 1975) of ' $L_{\alpha}$ ' peaks of $\mathrm{La}$ and $\mathrm{Nd}$ give the clue that both may have approximately the same affinity to their oxalate formation. The XPS results too, support well that $\mathrm{La}$ and $\mathrm{Nd}$ are present in the sample, besides the existence of these elements in their oxide states (figures $9-10$ ), especially $\mathrm{La}$ and $\mathrm{Nd}$ getting linked to $\mathrm{O}^{-}$of $\left(\mathrm{C}_{2} \mathrm{O}_{4}\right)^{2-}$. These results are in excellent agreement with the proposed empirical structure for LNO (figure 1).

The 'fogginess' of LNO (figure 2a) grown nearer to the precipitate layer is attributed to the fast diffusion rate of the upper reactant, by which the 'gel inclusion' is feasible and possible. At greater depths, meaning lower diffusion rates, gel inclusion becomes negligible, thereby making LNO crystals grown at greater depths very 'clear' (figure 2b). EDAX and XPS of 'foggy' crystals support the above explanation, by the revelation of $\mathrm{Si}\left(K_{\alpha}\right)$ and $(2 p) \mathrm{SiO}_{2}$, respectively. The peak relating to $\mathrm{Si}\left(K_{c t}\right)$ is totally absent in the EDAX of clear crystals (dashed line in figure $8 \mathrm{~b}$ ), thereby further confirming that 'gel inclusion' is 'nil' or 'negligible'.

\section{Conclusion}

It is concluded that the gel grown LNO sample reported in the present work (i) is ordered and crystalline as evidenced by the formation of well defined tabular crystals having hexagonal basal planes, (ii) is single crystalline in nature (XRD), (iii) accounts for the presence of oxalato complex by the revelation of their characteristic peaks (IR), (iv) loses $\mathrm{CO}$ and $\mathrm{CO}_{2}$ around $400^{\circ} \mathrm{C}$ (TGA), (v) has La and $\mathrm{Nd}$ established qualitatively and to some extent quantitatively (EDAX), (vi) is having La and $\mathrm{Nd}$ in their oxide states especially getting linked to $\mathrm{O}^{-}$of oxalate group (XPS) and (vii) yield 'gel inclusion' free clear crystals at greater depths, meaning thereby, better quality good crystals than the 'foggy' crystals.

\section{Acknowledgements}

Authors thank the Chairman, Materials Research Centre, Indian Institute of Science, Bangalore for XRD and IR, and Regional Sophisticated Instrumentation Centre, Indian Institute of Technology, Madras, for TGA and XPS facilities.

\section{References}

Barkley J R, Brixner L H and Hogan E M 1971 IEEE symposium on the applications of ferroelectrics (New York: York Town Heights)

Barkley J R, Brixner L H, Hogan E M and Waring R K 1972 J. Ferroelectrics 3191

Beille J et al 1987 C. R. Acad. Sci. Ser. 23041097

Brixner L H 1973 J. Cryst. Growth 18297

Caillaud F, Baumard J-F and Smith A 1988 Mater. Res. Bull. 231273

Eyring L 1964 Progress in science and technology (New York: Pergaman Press) 1/2 416

Henisch H K 1970 Crystal growth in gels (Pennsylvania: Pennsylvania University Press)

Henisch H K, Dennis J and Hanoka J I 1965 Phys. Chem. Solids 26493

Huheey J E 1983 Inorganic chemistry-Principles of structure and reactivity (New York: Harper International) 3rd ed. p. 802

Jayakumari Isac, Ittyachen M A and Raju K S 1995 Bull. Mater. Sci. 19495

Pacter A 1977 Krist \& Tech. 12729

Raju K S, Krishna K N, Jayakumari Isac and Ittyachen M A 1994 Bull. Mater. Sci. 171447

Riggs W M and Parker M J 1975 Methods of surface analysis (ed.) A W Czanderna (Amsterdam: Elsevier Scientific Pub. Co.) Ch. 4, p. 108

Sapriel J and Vacher R 1977 J. Appl. Phys. 481191

Sushma Bhat, Kotru P N and Raju K S 1995 Curr. Sci. 69 607

Wagner C D, Riggs W M, Davis L E, Moulder J F and Mullenberg J E (eds) 1978 Handbook of X-ray photoelectron spectroscopy (Minnesota: Perkin Elmer) 